\title{
Putative Digenic GJB2/MYO7A Inheritance of Hearing Loss Detected in a Patient with 48,XXYY Klinefelter Syndrome
}

\author{
Qin Zhang Tiantian Qin Wenmu Hu Muhammad Usman Janjua Ping Jin
}

Department of Endocrinology, The Third Xiangya Hospital, Central South University, Changsha, China

\section{Keywords}

Klinefelter syndrome · 48,XXYY syndrome · MYO7A · GJB2 •

Digenic inheritance $\cdot$ Nonsyndromic hearing loss

\begin{abstract}
Objectives: Nonsyndromic hearing loss (NSHL) is the most frequent type of hereditary hearing impairment. Here, we explored the underlying genetic cause of NSHL in a threegeneration family using whole-exome sequencing. The proband had concomitant NSHL and rare 48,XXYY Klinefelter syndrome. Material and Methods: Genomic DNA was extracted from the peripheral blood of the proband and their family members. Sanger sequencing and pedigree verification were performed on the pathogenic variants filtered by whole-exome sequencing. The function of the variants was analyzed using bioinformatics software. Results: The proband was digenic heterozygous for p.V37I in the GJB2 gene and p.L347I in the MYO7A gene. The proband's mother had normal hearing and did not have any variant. The proband's father and uncle both had NSHL and were compound for the GJB2 p.V37I and MYO7A p.L347I variants, thus indicating a possible GJB2/MYO7A digenic inheritance of NSHL. 48,XXYY Klinefelter syndrome was discovered in the proband after
\end{abstract}

karger@karger.com www.karger.com/hhe

Karger $\stackrel{\text { ' }}{5}$
(C) 2021 The Author(s)

Published by S. Karger AG, Basel

This is an Open Access article licensed under the Creative Common Attribution-NonCommercial-4.0 International License (CC BY-NC) (http://www.karger.com/Services/OpenAccessLicense), applicable to the online version of the article only. Usage and distribution for commercial purposes requires written permission. the karyotype analysis, while his parents both had normal karyotypes. Conclusions: Our findings reported a putative GJB2/MYO7A digenic inheritance form of hearing loss, expanding the genotype and phenotype spectrum of NSHL. In addition, this is the first report of concomitant NSHL and $48, \mathrm{XXYY}$ syndrome.

C 2021 The Author(s)

Published by S. Karger AG, Basel

\section{Introduction}

Hearing loss (HL) is one of the most common prevalent sensory defects in humans with a frequency of 1-3 per 1,000 newborns, and more than $50 \%$ of these cases are attributed to genetic factors [1]. HL has two major types: syndromic HL (SHL) and nonsyndromic HL (NSHL). NSHL is genetically heterogeneous and has various modes of transmission, including autosomal recessive, autosomal dominant, $\mathrm{X}$-linked, and mitochondrial deafness [2]. Currently, more than 100 genes are associated with NSHL (see Hereditary Hearing Loss homepage at http://hereditaryhearingloss. org). Among them, the GJB2, SLC26A4, MYO7A, OTOF, and $\mathrm{CDH} 23$ genes are commonly found. With the increased access to next-generation sequencing, the simultaneous de-

Correspondence to:

Ping Jin, ping.jin06@csu.edu.cn 
tection of variants in two or more genes in patients with NSHL is being discovered, paving a way to the possibility of digenic/oligogenic inheritance of the disease.

48, XXYY Klinefelter syndrome is a rare type of Klinefelter syndrome, which was first described by Muldal et al. [3]. To date, more than 100 cases with 48,XXYY Klinefelter syndrome have been reported, with the incidence of $1 / 18,000-1 / 5,000$ male births [3]. However, no study has reported on congenital HL combined with 48,XXYY Klinefelter syndrome. In this study, we identified a novel digenic GJB2/MYO7A inheritance of HL in a three-generation family affected by NSHL using whole-exome sequencing. The proband had concomitant NSHL and rare 48,XXYY Klinefelter syndrome.

\section{Patients and Methods}

\section{Family Enrollment and Clinical Evaluation}

We studied a three-generation Chinese family with four members affected by congenital HL (Fig. 1a). The proband had concomitant NSHL and 48,XXYY Klinefelter syndrome. Hearing status was determined by self-assessment or pure-tone audiometry (PTA). PTA was calculated as the average of the hearing level at $0.5,1.0,2.0$, and $4.0 \mathrm{kHz}$. The level of HL, in terms of PTA, was described as follows: normal hearing, $<25 \mathrm{~dB}$; mild hearing impairment, 26-40 dB; moderate hearing impairment, 41-60 dB; severe hearing impairment, $61-80 \mathrm{~dB}$; and profound hearing impairment, $>81 \mathrm{~dB}$. The Romberg test and tandem-walking test were performed to assess the vestibular function. Ocular examination included slit-lamp examination and stereoscopic fundoscopy.

\section{Ethical Statement}

Peripheral blood samples were collected from the family and from 100 unrelated healthy controls. DNA was isolated from the peripheral blood by phenol-chloroform procedures. The Institutional Ethics Committee of the Third Xiangya Hospital approved this study. Written informed consent was obtained from all subjects enrolled in this study. All procedures were performed according to the World Medical Association's Declaration of Helsinki.

\section{Methods}

Whole-Exome Sequencing

Genomic DNA was isolated from the peripheral blood by standard phenol-chloroform procedures. The isolated DNA was sheared on a Bioruptor UCD-200 (Diagenode, Denville, NJ, USA). For all samples, shearing worked consistently, and the size distribution peak was approximately $200 \mathrm{bp}$. DNA libraries were prepared using the KAPA Library Preparation Kit (KR0453, Kapa Biosystems, Wilmington, MA, USA). The whole-exome sequencing was performed using SureSelectXT2 Target Enrichment System (Agilent Technologies, Santa Clara, CA, USA). Sample dilution, flow cell loading, and sequencing were performed according to Illumina's specifications. DNA libraries were sequenced on the HiSeq2500 platform (Illumina, San Diego, CA, USA) as paired-end 200-bp reads. Raw data were filtered and aligned against the human reference genome (hg19) using BWA Aligner (http://bio-bwa.sourceforge.net/). Single-nucleo- tide polymorphisms were identified using Genome Analysis Toolkit (www.broadinstitute.org/gatk). Variants were annotated using ANNOVAR (annovar.openbioinformatics.org/en/latest/). We anticipated that a causative variant would be missense or gene-disrupting and would be rare in the overall population. Selected variants were checked in relevant variant frequency databases (ESP, dbSNP, 1000 Genomes, ClinVar, and HGMD). We applied filtering criteria that required a variant to have a frequency of $<1 \%$ in the 1000 Genomes, ExAC, and gnom $A D$ databases. In addition, variations that can cause clinical symptoms were further analyzed. All variants were interpreted according to the standards set out by the American College of Medical Genetics (ACMG) and categorized as pathogenic, likely to be pathogenic, variants of uncertain significance, likely to be benign, and benign.

\section{Sanger Sequencing}

The candidate variants identified by whole-exome sequencing were then confirmed by polymerase chain reaction (PCR). Primer sequences used for PCR amplification and DNA sequencing of the gap junction protein beta 2 (GJB2) gene (NM_004004) exon 2 were as follows: $5^{\prime}$-GAGCCTTGACAGCTGAGCA-3' and $5^{\prime}$-TGGCCTACCGGAGACATGA-3'; those of the myosin VIIA (MYO7A) gene (NM_00112718) exon 10 were as follows: 5'-TGCCTGCAGACTCATGGTG-3' and 5'-CACAGGACCTGCACATGGT- $3^{\prime}$. Variants were identified by direct sequencing of PCR products bidirectionally using an ABI 3730xl automated sequencer (Applied Biosystems, USA). Segregation analysis of the respective $M Y O 7 A$ and GJB2 pathogenic variants was performed within the families.

\section{Results}

\section{Clinical Characteristics}

The proband (Fig. 1a: III-1) was a 27 -year-old male who was referred to our hospital due to HL and the absence of pubertal development. Physical examination showed a height of $170 \mathrm{~cm}$, a weight of $36 \mathrm{~kg}$, and a Tanner developmental stage of G2P1. Laboratory tests showed low levels of testosterone $(102.7 \mathrm{ng} / \mathrm{dL}$; reference range [RR] 249-836 ng/dL) and high levels of follicle-stimulating hormone (97.53 mIU/mL; RR 1.7-8.6 mIU/mL) and luteinizing hormone (43.19 mIU/mL; RR 1.7-8.6). Ultrasound examination showed small testicular volumes (left, $15 \times 7 \mathrm{~mm}$; right, $15 \times 8 \mathrm{~mm}$ ). No dysmorphic facial abnormalities were observed, such as ocular hypertelorism

Fig. 1. Illustration of the family with digenic GJB2/MYO7A variants. The pedigree shows mutations in GJB2/MYO7A genes (a). Chromosome analyses of the proband revealed 48 ,XXYY (b). The different hearing levels and the genotype-phenotype relation in the family members are summarized (c). Sequencing chromatograms of the GJB2 p.V37I and MYO7A p.L347I variants (d).

(For figure see next page.) 

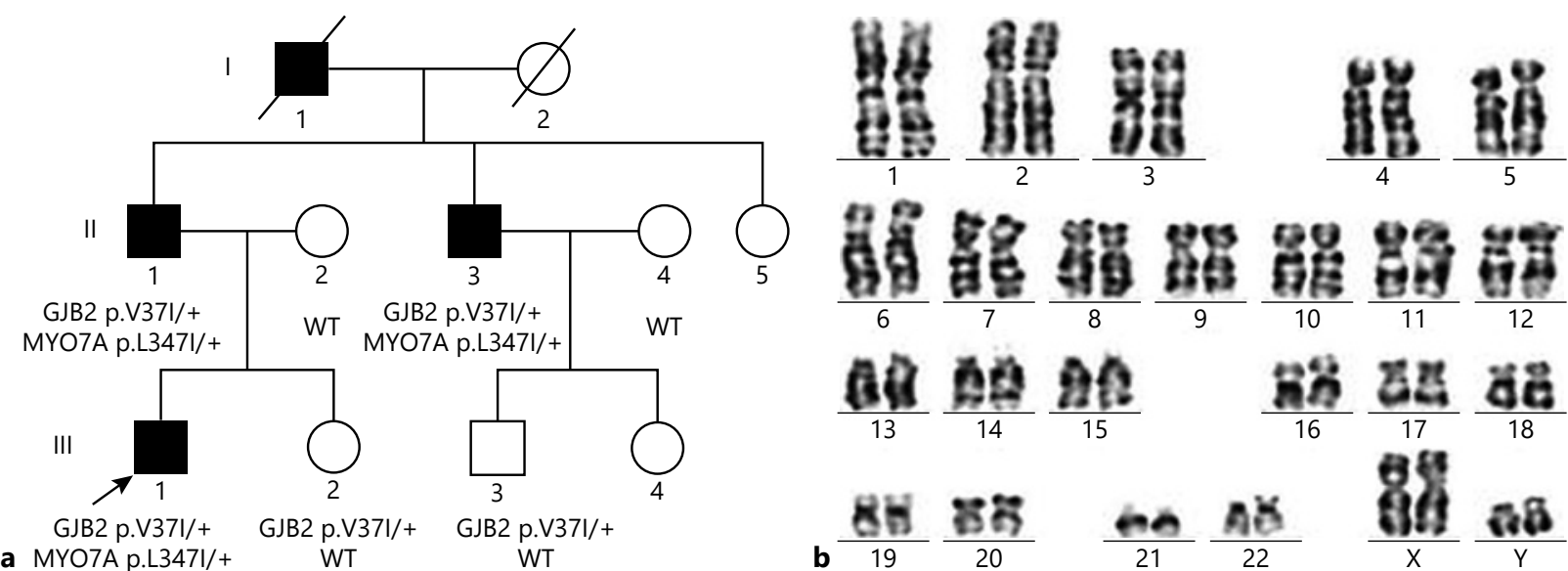

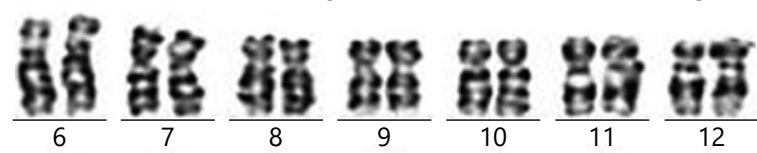

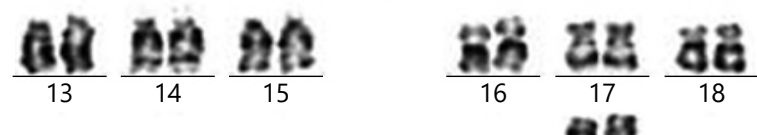

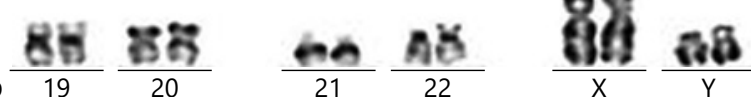
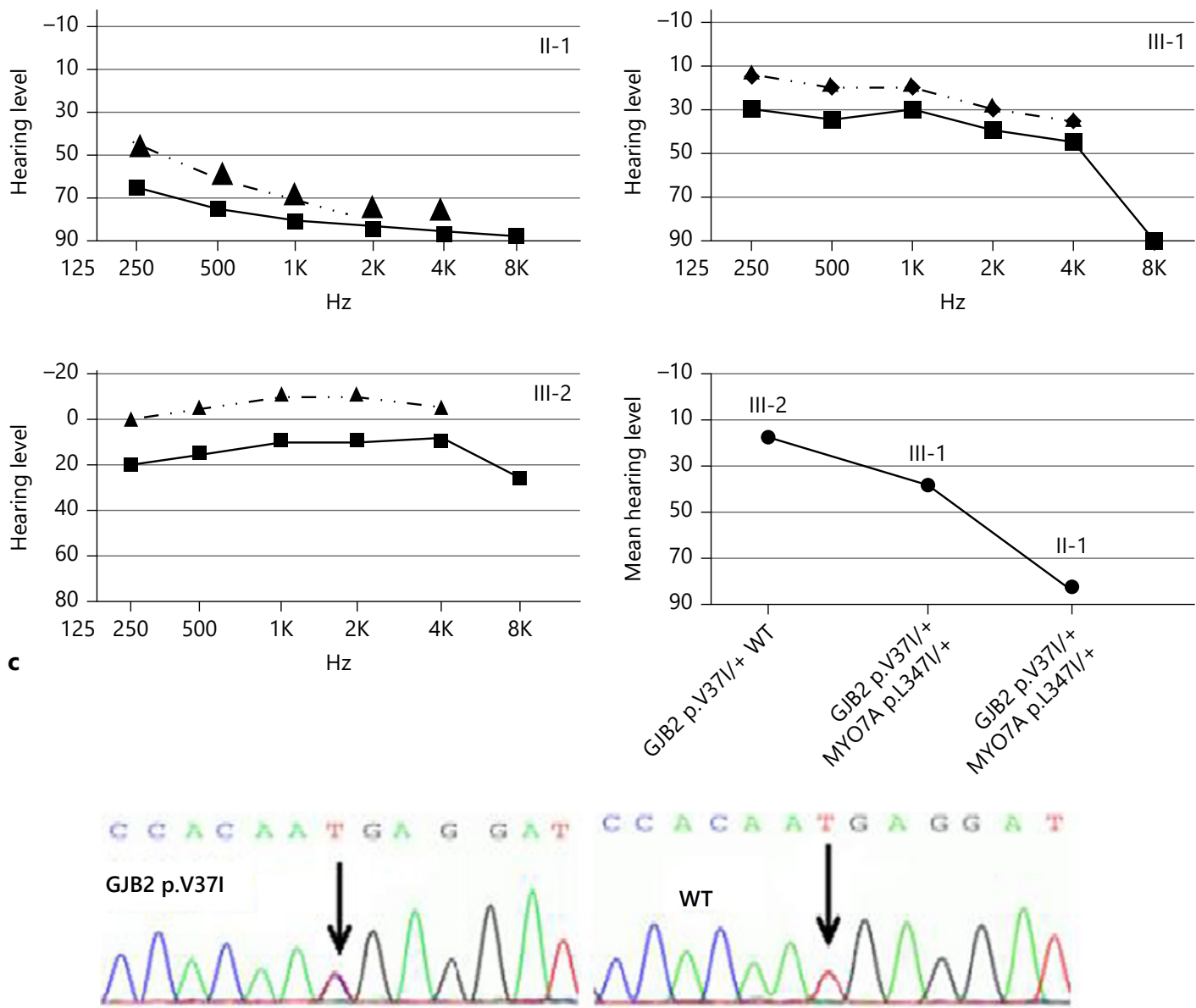

$\mathrm{G} G A \mathrm{~A} A \mathrm{~A}$ G A G G A T
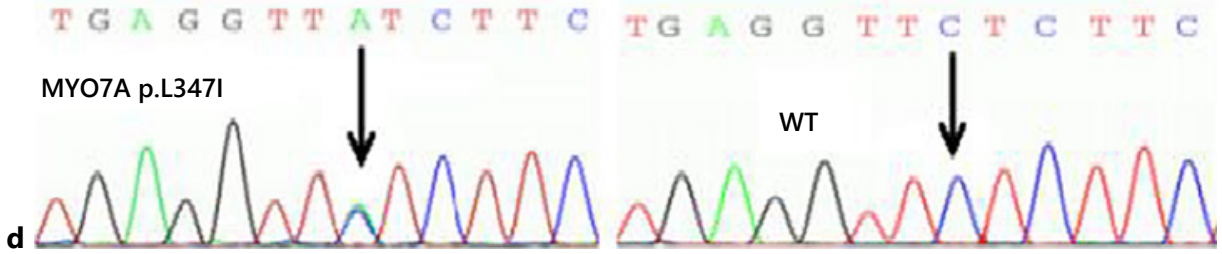


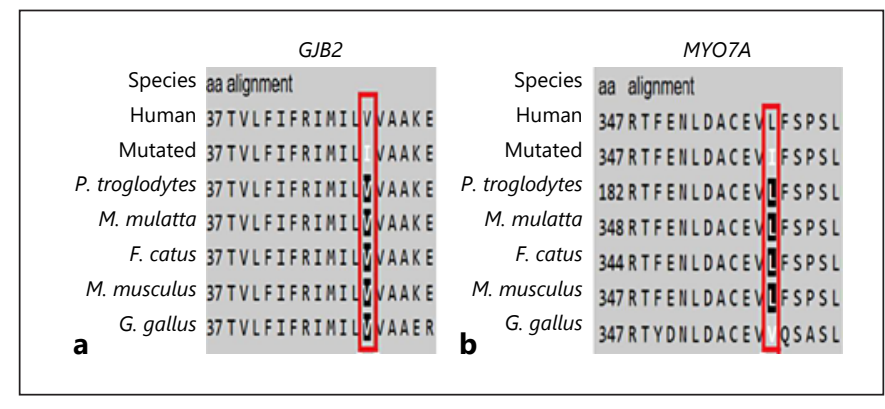

Fig. 2. Multiple alignment of the GJB2, MYO7A protein sequence in different species, indicating conservation of the residues GJB2 p.Val37 (a), and MYO7A p.Leu347(b) affected by these mutations.

and pes planus. The patient had delayed speaking and learning difficulties. His IQ was only 70. Karyotype analysis showed a chromosomal composition of 48,XXYY (Fig. 1b), which indicated Klinefelter syndrome. The parents of the proband were not consanguineously married and had normal karyotypes. The proband and his father (Fig. 1a: II-1), uncle (Fig. 1a: II-3), and grandfather (Fig. 1a: I-1) had HL since childhood; however, all had never received hearing aids. PTA demonstrated mild bilateral sensorineural HL in the proband, and severe bilateral sensorineural HL in his father (Fig. 1c). The proband's sister (Fig. 1a: III-2), mother (Fig. 1a: II-2), and cousins (Fig. 1a: III-3, 4) had self-reported normal hearing. The audiological evaluation of the proband's sister disclosed normal hearing (Fig. 1c). No vestibular dysfunction or retinal defects were observed in the family members.

\section{Variants Identified by Whole-Exome Sequencing}

The proband (Fig. 1a: III-1) was compound heterozygous for the c.109G $>\mathrm{A}$ (p.V37I) variant in the GJB2 gene and the c.1039C $>$ A (p.L347I) variant in the MYO7A gene (Fig. 1d). His father (Fig. 1a: II-1) and uncle (Fig. 1a: II-3), who also had congenital deafness, were both digenic heterozygote for the p.V37I variant in the GJB2 gene and the p.L347I variant in the MYO7A gene. The proband's mother (Fig. 1a: II-2) did not carry the p.V37I or p.L347I variant. His sister (Fig. 1a: III-2) and cousin (Fig. 1a: III3 ) were both heterozygous for the p.V37I variant in the GJB2 gene. The prevalence of GJB2 p.V37I and MYO7A p.L347I variants were examined in 100 healthy individuals. Among the 100 healthy individuals, $11(11.0 \%)$ were heterozygous for the GJB2 p.V37I variant and none were heterozygous for the MYO7A p.L347I variant. The $\mathrm{p}$. V37I/p.L347I variants and GJB2/MYO7A digenic inheritance were not detected in the 100 healthy individuals.

\section{In silico Analysis}

The GJB2 missense variant p.V37I has been reported previously and was predicted to be damaging using PolyPhen-2 and MutationTaster and tolerable using SIFT and PROVEAN. Amino acid alignment of GJB2 among different species revealed that the valine mutated at position 37 was conserved among all species examined (Fig. 2a). According to ACMG guidelines, the GJB2 p.V37I variant was interpreted as pathogenic since this variant has been reported as a pathogenic variant (PS1), with extremely low frequency (PM2), and was predicted to be damaging and disease-causing using PolyPhen-2 and MutationTaster (PP3).

The missense MYO7A p.L347I variant was predicted to be disease-causing using SIFT, benign using PolyPhen-2, and tolerable using MutationTaster and PROVEAN. Amino acid alignment of MYO7A among different species revealed that the leucine mutated at position 347 was conserved among species examined (Fig. 2b). According to ACMG guidelines, the MYO7A p.L347I variant was interpreted as variant of uncertain significance since this variant has an extremely low frequency (PM2) and was predicted to be damaging and disease-causing using SIFT (PP3). The MYO7A p.L347I variant was not found in HGMD (http:// www.hgmd.cf.ac.uk/), dbSNP (http://www.ncbi.nlm.nih. gov/snp), and ClinVar (http://www.ncbi.nlm.nih.gov/clinvar), indicating that it was a novel variant.

\section{Discussion}

Most NSHL forms are monogenic and have genetic heterogeneity. However, digenic/oligogenic inheritance has been reported recently [4-9]. Here, the novel compounds heterozygous for the GJB2 p.V37I variant and MYO7A p.L347I variant were found in the proband. The GJB2 gene accounts for approximately $50 \%$ of nonsyndromic autosomal recessive HL (DFNB2) in different populations [10, 11]. The GJB2 gene encodes the gap junction subunit protein connexin 26 ( $\mathrm{Cx} 26)$, which plays a crucial role in intercellular communication by forming the cochlear gap junction. In the inner ear, GJB2 plays a vital role in many auditory processes, including potassium recycling, energy supply, and maintenance of endolymphatic homeostasis $[12,13]$. Currently, more than 300 GJB2 variants are associated with HL (HGMD, http://www.hgmd.cf.ac.uk/ac/). The GJB2 p.V37I variant has a high allele frequency (up to $10 \%)$ in East Asians $[10,14,15]$. However, the pathogenicity of the p.V37I has yet to be elucidated. Early studies have shown the p.V37I variant as a benign polymorphism, as it was observed in unaffected heterozygous controls. How- 
Table 1. Reported digenic and trigenic inheritance in NSHL

\begin{tabular}{|c|c|c|c|c|}
\hline Patient No. & Variant 1 & Variant 2 & Variant 3 & Reference \\
\hline 1 & GJB2 c. $109 \mathrm{G}>\mathrm{A}$ & GJB2 c.638T>C & \multirow[t]{9}{*}{ GJB3 c. $250 \mathrm{G}>\mathrm{A}$} & Chen et al. [4] \\
\hline 2 & GJB2 с.638T>C & GJB3 c. $250 \mathrm{G}>\mathrm{A}$ & & Chen et al. [4] \\
\hline 3 & GJB2 c. $235 \mathrm{delC}$ & GJB3 c. $497 \mathrm{G}>\mathrm{A}$ & & Liu et al. [5] \\
\hline 4 & GJB2 c.235delC & GJB3 c.580G $>\mathrm{A}$ & & Liu et al. [5] \\
\hline 5 & GJB2 c.299delAT & GJB3 c.580G $>A$ & & Liu et al. [5] \\
\hline 6 & GJB2 c.109G >A & GJB3 c. $580 \mathrm{G}>\mathrm{A}$ & & Chen et al. [6] \\
\hline 7 & GJB2 c.578T >A & $G J B 3$ c. $580 \mathrm{G}>\mathrm{A}$ & & Kim et al. [7] \\
\hline 8 & GJB2 c. $235 \mathrm{delC}$ & MITF c. $1021 \mathrm{C}>\mathrm{T}$ & & Kim et al. [7] \\
\hline 9 & GJB2 c. $487 \mathrm{~A}>\mathrm{G}$ & TMPRSS3 c.895G $>\mathrm{A}$ & & Leone et al. [8] \\
\hline 11 & GJB2 c.35delG & TMPRSS3 c. $208 \mathrm{delC}$ & \multirow[t]{2}{*}{ TMPRSS3 c.579dupA } & Battelino et al. [9] \\
\hline 12 & GJB2 c.109G $>A$ & MYO7A c. $1039 \mathrm{C}>\mathrm{A}$ & & Present study \\
\hline
\end{tabular}

ever, an increasing body of evidence has indicated that both homozygotes and compound heterozygous for the p.V37I variant were associated with mild to moderate hearing impairment $[16,17]$.

The MYO7A gene encodes the actin-based motor protein myosin-VIIa, which is especially crucial for the function of cochlear hair cells and eye development [18]. The myosin VIIa protein contains a conserved N-terminal actin-binding and ATPase domain (motor domain), a neck region containing five isoleucine-glutamine (IQ) motifs, and a short predicted coiled-coil domain, followed closely by two myosin tail homology 4 (MyTH4) domains, two band 4.1-ezrin-radixin-moesin (FERM) domains, and an SH3 domain [18]. The MYO7A gene has long been associated with Usher syndrome type 1B (USH1B), which is characterized by sensorineural HL, retinitis pigmentosa, and vestibular dysfunction $[18,19]$. In 1997, Weil et al. [20] and Liu et al. [21] have identified that MYO7A gene variants are associated with DFNB2. So far, most variants identified in MYO7A are associated with USH1B, whereas only approximately 17 MYO7A variants have been found to be responsible for DFNB2 $[22,23]$. The novel MYO7A missense p.L347I variant located in the motor domain was predicted to be damaging using in silico analysis. The absence of vestibular and retinal defects in the affected patients suggests that this family has an isolated NSHL presentation, instead of USH1B.

It is estimated that $6-20 \%$ of GJB2 mutations in subjects with HL were monoallelic mutations [24]. Researchers have hypothesized that a single heterozygous GJB2 mutant was possibly contributing to deafness via digenic inheritance. In 2009, Liu et al. [5] have identified heterozygous GJB2 and GJB3 mutations in patients with NSHL. Since then, many other forms of digenic inheritance have

Digenic GJB2/MYO7A Inheritance of

Hearing Loss in 48,XXYY Syndrome been reported [4-9], such as GJB2/GJB3, GJB2/MITF, and GJB2/TMPRSS3 (Table 1). Although digenic inheritance has been increasingly described in NSHL, its prevalence is unknown. Chen et al. [4] have assessed the contributions of variants in GJB3 or GJB6 in a cohort of 100 patients with NSHL with likely pathogenic heterozygous $G J B 2$ mutations. Putatively causative GJB3 variants were $1 \%$, and no GJB6 mutation was found. Furthermore, Ołdak et al. [25] have screened GJB2 variants in 42 patients with HL with at least one TMPRSS3 pathogenic variant and identified four individuals who were double heterozygous for pathogenic GJB2 and TMPRSS3 variants. They have proposed that the contributions of GJB2 digenic inheritance may not be predominant. In this study, the proband and his father and uncle were digenic heterozygous for the MYO7A p.L347I and GJB2 p.V37I variants. Based on the co-segregated analysis, we implied a possible novel GJB2/MYO7A digenic inheritance of NSHL in this family. However, this study has some limitations. First, it relies exclusively on data from only one family. Second, in vitro data demonstrating a functional link between these two genes are missing. Third, the dominant character of the identified GJB2 p.V37I variant with a possible incomplete penetrance in the family cannot be excluded. Further animal models or cell biology experiments and studies with a large sample size remain needed to clarify the role of the digenic mutations involved.

In this study, the proband had concomitant NSHL and rare 48,XXYY Klinefelter syndrome. Compared with 47 , XXY Klinefelter syndrome, 48, XXYY syndrome is associated with more severe neurodevelopmental and behavioral abnormalities [26-28]. Tartaglia et al. [26] have found that patients with 48 ,XXYY syndrome had some facial dysmorphic features, such as ocular hypertelorism, pes pla- 
nus, upward-slanting palpebral fissures, joint laxity, and dental problems. Other medical issues, including allergies and asthma, congenital heart defects, inguinal hernia, and cryptorchidism, were also more common in individuals with 48,XXYY syndrome. However, HL is rarely reported in patients with Klinefelter syndrome. In 1971, Anderson et al. [29] have studied the hearing ability of 26 men with different types of sex chromosome anomalies (usually 47,XXYY [Klinefelter syndrome]). They found that the number of hearing threshold impairments with a probably endogenous origin was unusually high (19\%). However, genetic testing was not performed. In 2019, Li et al. [30] have reported a case of concomitant Usher syndrome and Klinefelter syndrome (47,XXYY). To the best of our knowledge, the case reported here is the first reported case of concomitant NSHL and 48,XXYY Klinefelter syndrome in the literature. Whether the NSHL is a new phenotypic feature of 48,XXYY Klinefelter syndrome or it happened by accident needs further investigation.

In conclusion, we reported a putative GJB2/MYO7A digenic inheritance form of $\mathrm{HL}$ in a three-generation family affected by NSHL using whole-exome sequencing, expanding the genotype and phenotype spectrum of NSHL. In addition, this is the first report of a molecularly diagnosed case of concomitant NSHL and 48,XXYY Klinefelter syndrome.

\section{Acknowledgement}

We are grateful to all family members included in this study for their invaluable participation and cooperation.

\section{Statement of Ethics}

The study was conducted in accordance with the Declaration of Helsinki and approved by an independent Ethic Committee (The Institutional Review Board of Third Xiangya Hospital, Central South University, China).The reference number of this project is 2021-S165. The Institutional Ethics Committee of the Third Xiangya Hospital approved this study. Written informed consent was obtained from all subjects enrolled in this study.

\section{Conflict of Interest Statement}

The authors declare that they have no competing interests.

\section{Funding Sources}

This work was supported by the National Natural Science Foundation of China (81670730, 81100583), Natural Science Foundation of Hunan Province (2016JJ4103).

\section{Author Contributions}

Q.Z. performed the research and wrote the manuscript; T.Q., W.H., and M.U.J. coordinated the research; P.J. designed the research study and revised the manuscript. All authors read and approved the final manuscript.

\section{References}

1 Morton CC, Nance WE. Newborn hearing screening - a silent revolution. $\mathrm{N}$ Engl J Med. 2006 May;354(20):2151-64.

2 Van Camp G, Willems PJ, Smith RJ. Nonsyndromic hearing impairment: unparalleled heterogeneity. Am J Hum Genet. 1997 Apr;60(4):758-64.

3 Muldal S, Ockey CH, Thompson M, White LL. 'Double male' - a new chromosome constitution in the Klinefelter syndrome. Acta Endocrinol (Copenh). 1962 Feb;39(2):183203.

4 Chen K, Wu X, Zong L, Jiang H. GJB3/GJB6 screening in GJB2 carriers with idiopathic hearing loss: is it necessary? J Clin Lab Anal. 2018 Nov;32(9):e22592.

5 Liu XZ, Yuan Y, Yan D, Ding EH, Ouyang $\mathrm{XM}$, Fei Y, et al. Digenic inheritance of nonsyndromic deafness caused by mutations at the gap junction proteins Cx26 and Cx31. Hum Genet. 2009 Feb;125(1):53-62.

6 Chen K, Zong L, Zhou W, Liu M, Jiang H. GJB3 and GJB6 genes screening in 200 nonsyndromic hearing impairment patients. Audiol Speech Pathol. 2012;20:198-200.
7 Kim SY, Kim AR, Kim NK, Lee C, Kim MY, Jeon EH, et al. Unraveling of Enigmatic Hearing-Impaired GJB2 Single Heterozygotes by Massive Parallel Sequencing: DFNB1 or Not? Medicine(Baltimore).2016 Apr;95(14):e3029.

8 Leone MP, Palumbo P, Ortore R, Castellana S, Palumbo O, Melchionda S, et al. Putative TMPRSS3/GJB2 digenic inheritance of hearing loss detected by targeted resequencing. Mol Cell Probes. 2017 Jun;33:24-7.

9 Battelino S, Klancar G, Kovac J, Battelino T, Trebusak Podkrajsek K. TMPRSS3 mutations in autosomal recessive nonsyndromic hearing loss. Eur Arch Otorhinolaryngol. 2016;273(5):1151-4.

10 Dai P, Yu F, Han B, Liu X, Wang G, Li Q, et al. GJB2 mutation spectrum in 2,063 Chinese patients with nonsyndromic hearing impairment. J Transl Med. 2009 Apr;7(1):26.

11 Koohiyan M, Ahmadi A, Koohian F, Aghaei S, Amiri B, Hashemzadeh-Chaleshtori M. An update of spectrum and frequency of GJB2 mutations causing hearing loss in the south of Iran: A literature review. Int J Pediatr Otorhinolaryngol. 2019 Apr;119:136-40.
12 Liu YP, Zhao HB. Cellular characterization of Connexin 26 and Connnexin30 expression in the cochlear lateral wall. Cell Tissue Res. 2008 Sep;333(3):395-403.

13 Zhao HB, Yu N. Distinct and gradient distributions of connexin 26 and connexin 30 in the cochlear sensory epithelium of guinea pigs. J Comp Neurol. 2006 Nov;499(3):506-18.

14 Tsukada K, Nishio S, Usami S; Deafness Gene Study Consortium. A large cohort study of GJB2 mutations in Japanese hearing loss patients. Clin Genet. 2010 Nov;78(5):464-70.

$15 \mathrm{Wu}$ CC, Tsai CH, Hung CC, Lin YH, Lin YH, Huang FL, et al. Newborn genetic screening for hearing impairment: a population-based longitudinal study. Genet Med. 2017 Jan;19(1):6-12.

16 Chan DK, Chang KW. GJB2-associated hearing loss: systematic review of worldwide prevalence, genotype, and auditory phenotype. Laryngoscope. $2014 \mathrm{Feb} ; 124(2):$ :34-53.

17 Chai Y, Chen D, Sun L, Li L, Chen Y, Pang X, et al. The homozygous p.V37I variant of GJB2 is associated with diverse hearing phenotypes. Clin Genet. 2015 Apr;87(4):350-5. 
18 Wolfrum U, Nagel-Wolfrum K. Das UsherSyndrom, eine Ziliopathie des Menschen [The Usher Syndrome, a Human Ciliopathy]. Klin Monatsbl Augenheilkd. 2018 Mar;235(3):273-80.

19 He X, Peng Q, Li S, Zhu P, Wu C, Rao C, et al A novel mutation in the MYO7A gene is associated with Usher syndrome type 1 in a Chinese family. Int J Pediatr Otorhinolaryngol. 2017 Aug;99:40-3.

20 Weil D, Küssel P, Blanchard S, Lévy G, LeviAcobas F, Drira M, et al. The autosomal recessive isolated deafness, DFNB2, and the Usher $1 \mathrm{~B}$ syndrome are allelic defects of the myosinVIIA gene. Nat Genet. 1997 Jun;16(2):191-3.

21 Liu XZ, Walsh J, Mburu P, Kendrick-Jones J, Cope MJ, Steel KP, et al. Mutations in the myosin VIIA gene cause non-syndromic recessive deafness. Nat Genet. 1997 Jun;16(2):18890.
22 Ben-Salem S, Rehm HL, Willems PJ, Tamimi ZA, Ayadi H, Ali BR, et al. Analysis of two Arab families reveals additional support for a DFNB2 nonsyndromic phenotype of MYO7A. Mol Biol Rep. 2014 Jan;41(1):193200.

23 Li Y, Su J, Ding C, Yu F, Zhu B. Identification of four novel mutations in MYO7A gene and their association with nonsyndromic deafness and Usher Syndrome 1B. Int J Pediatr Otorhinolaryngol. 2019 May;120:166-72.

24 Hutchin T, Coy NN, Conlon H, Telford E, Bromelow K, Blaydon D, et al. Assessment of the genetic causes of recessive childhood nonsyndromic deafness in the UK - implications for genetic testing. Clin Genet. 2005 Dec;68(6):506-12.

25 Ołdak M, Lechowicz U, Pollak A, Oziębło D, Skarżyński H. Overinterpretation of high throughput sequencing data in medical genetics: first evidence against TMPRSS3/GJB2 digenic inheritance of hearing loss. J Transl Med. 2019 Aug;17(1):269.
26 Tartaglia N, Davis S, Hench A, Nimishakavi S, Beauregard R, Reynolds A, et al. A new look at XXYY syndrome: medical and psychological features. Am J Med Genet A. 2008 Jun;146A(12):1509-22.

27 Tartaglia N, Ayari N, Howell S, D'Epagnier C, Zeitler P. 48,XXYY, 48,XXXY and 49,XXXXY syndromes: not just variants of Klinefelter syndrome. Acta Paediatr. 2011 Jun;100(6):851-60.

28 Hanley AP, Blumenthal JD, Lee NR, Baker EH, Clasen LS, Giedd JN. Brain and behavior in $48, \mathrm{XXYY}$ syndrome. Neuroimage Clin. 2015 Apr;8:133-9.

29 H Anderson, J Lindsten, E Wedenberg. Hearing defects in males with sex chromosome anomalies. Acta Otolaryngol. 1971;72(1):558.

30 Li X, Huang S, Yuan Y, Lu Y, Zhang D, Wang $\mathrm{X}$, et al. Detecting novel mutations and combined Klinefelter syndrome in Usher syndrome cases. Acta Otolaryngol. 2019 Jun;139(6):479-86. 